\title{
Quantum algorithms for computational nuclear physics
}

\author{
Jakub Višňák $1,2,3, a$ \\ ${ }^{1}$ Dep. of Nuclear Chemistry, FNSPE, Czech Technical University, Břehová 7, 11519 Prague 1, Czech Rep. \\ ${ }^{2}$ Dep. of Chemical Phys. and Optics, Faculty of Mathematics and Phys., Charles University, Ke Karlovu 3, 12116 Prague 2, Czech Rep. \\ 3. J. Heyrovský Institute of Physical Chemistry, Dolejškova 2155/3, 18223 Prague 8, Czech Rep.
}

\begin{abstract}
While quantum algorithms have been studied as an efficient tool for the stationary state energy determination in the case of molecular quantum systems, no similar study for analogical problems in computational nuclear physics (computation of energy levels of nuclei from empirical nucleon-nucleon or quark-quark potentials) have been realized yet. Although the difference between the above mentioned studies might seem negligible, it will be examined. First steps towards a particular simulation (on classical computer) of the Iterative Phase Estimation Algorithm for deuterium and tritium nuclei energy level computation will be carried out with the aim to prove algorithm feasibility (and extensibility to heavier nuclei) for its possible practical realization on a real quantum computer.
\end{abstract}

\section{Introduction}

The Quantum computer as a model of information processing device exploiting directly the exclusively quantum resources - superpositioning, parallelism, entanglement and destructive interference [1-3] is a promissive tool for breaking the limits of asymptotical computational costs derived for classical algorithms for classical computers. Arguably there could be quantum algorithms (qA, i.e., algorithm tailored for quantum computer) with asymptotic complexity (in practical words - the time cost as a function of problem size) scaling significantly slower (most important case is polynomial scaling vs. exponential scaling) than the best possibly existing classical algorithm (for the same problem). The latter prediction is widely believed [1] (for opposing view see [4]), but still unproven [3]. However, there does exist a class of tasks which should prefer quantum computer over the classical one by their very nature - tasks of modelling quantum systems [5]. The other interesting problems for which are known qAs outperforming the best currently known classical algorithms are, e.g. Deutsch-Jozsa [6,7], Grover's [8] and the famous Shor's Algorithm [9] for integer primefactorization ${ }^{\mathrm{a}}$.

This study aims to prepare the first step towards the first simulation of a quantum algorithm for nuclear structure computation on a classical computer.

${ }^{a}$ Which, if successfully implemented on a large-scale quantum computer would make the current encrypting system RSA obsolete (on the other hand, quantum teleportation can provide us by fundamentally uninterceptable cryptographic method $[1,10,11])$
Simulations of this kind provide us answers on questions such as - "Which algorithm with which parameters is best for the class of computational problems in question? How many qubits will be needed to perform the algorithm? And how many quantum logical gates will be needed? (and therefore for how long will one computation take? Wouldn't be computation affected by decoherence after that time?)". The inspiration was taken from successful simulation studies done for the ab initio structure theory computational problems, i.e. Abrams and Lloyd Algorithm [12-16], its application to the $\mathrm{H}_{2}$ dissociation curve by Full-CI (Full Configuration Interaction method) in minimal basis [17] (including practical realization on photonic quantum computer), to slightly larger systems like $\mathrm{LiH}, \mathrm{H}_{2} \mathrm{O}$ [13] and $\mathrm{CH}_{2}$ [16], generalization for explicitly relativistic all electron 4component calculations via Dirac-Coulomb(-Breit) Hamiltonian for $\mathrm{SbH}$ model molecule [18] (the latter two were just simulations of algorithms on a classical computer) or another interesting algorithms, among them, the Quantum Variational Hamiltonian Estimation [56] applied on $\mathrm{HeH}^{+}$molecule electronic energy (for practical realization of $\mathrm{HeH}^{+}$energy calculation, please see [60]) should be cited as an example.

The nuclear structure problems, unlike the electronic structure ones, are complicated not only by computational complexity of many-body stationary Schroedinger equation but by uncertainty in choosing the correct Hamiltonian $^{\mathrm{b}}$ too. However, with the phenomenological b (in the case of mechanical model - the correct
description of either nucleon-nucleon or quark-quark
potentials ("quark potential" in this article refers always
to the effective phenomenological formulae based on the

\footnotetext{
a Corresponding author: jvisnak@gmail.com
} 
nucleon-nucleon potentials derived from the scattering experiments together with three-body (or eventually fouror more- body forces) carefully fitted to few model nuclei bonding energies do produce in principle numerically accurate Hamiltonian for small and medium nuclei and (sooner or later) the main bottleneck for nuclear structure modeling will turn out to be the diagonalization of Hamiltonian in Hilbert space of large dimension (scaling roughly as $n^{A}$, where $n$ is the one-particle basis set cardinality and $A$ is number of nucleons, therefore exponentially in $A$ ). Therefore, the same class of qAs as in the electronic structure may be applied.

Similarly as for the many-body nucleon model above, we can think about phenomenological mechanical model of nuclei as bounded state of $3 A$ (constituent, valence) quarks with given quark-quark effective potentials (the correspondence with the nucleonic model can be derived through Composite Particle Representation Theory [19], the feasible example of quark-quark potentials were derived by $\mathrm{Wu}[19])$.

Diagonalization of second quantized Hamiltonians ${ }^{\mathrm{c}}$ non-conserving the number of particles or their generalizations built from creation and annihilation operators for more then one kind of particles and containing terms corresponding for creation of multiple (anti)quarks or composite particles, etc. is probably completely intractable $^{\mathrm{d}}$ on classical computer. However, the Abrams-Lloyd algorithm via the direct-mapping vector space and algebra isomorphism can solve this problem in time polynomial in $n$ (the exact computational time cost is of form $\mathrm{O}\left(n^{a} \log ^{b} n\right)$, where I will discuss the $a$ and $b$ values for different algorithms below) and the qubit cost is of form $\mathrm{O}(n)$ ). Compact mapping [20] and expectation value averaging quantum algorithms provide similar speed up with respect to classical computer algorithm design - the number of quantum gates required and therefore the computational time scales as the number of terms in second quantized Hamiltonian or with overhead at most polynomial in $n$.

\section{Theoretical background}

\subsection{Quantum computer, qubits, gates}

For the sake of this article, we can think about quantum computer (here only the digital quantum computer will be presented) as an theoretical device composed of quantum

very simplified model of particles build just from the "constituent" quarks), however, since the kinetic energies of constituent particles are much higher than in the electronic case, the feasibility of any mechanical approach is in question)

c Through the Dirac picture and second quantized Hamiltonian (for qA simulation for electronic structure problem please note [18]) we can arrive into formulation similar to field-theory (going beyond the no-pair approximation [21]) with second quantized Hamiltonian consisting of terms creating particle-anti-particle pairs.

${ }^{\mathrm{d}}$ For realistically short computational time. Except for the smallest possible systems. core from $m$ distinguishable 2-level quantum systems (qubits), universal sets of gates (acting on the quantum core state as unitary operators) and measurement operators (measuring the state of defined subsets of qubits (quantum registers)) and classical control unit which realizes which gate or measurement should be applied according to program corresponding to particular algorithm. The computation result is decided upon the measurement outcomes. The computational model is stochastic and will produce the correct result with some probability ("the success probability", $p$ ). In case $p>1 / 2$ one can always increase the correct result probability as close to 1 as needed by repeating the computation enough times and by majority voting on the result.

In the whole article, the ideal digital quantum computer is addressed. Real quantum computers suffer from quantum noise and decoherence. In most cases the resulting negative effects on quantum computation can be mitigated by quantum error correcting codes [1, 22, 23].

\subsection{Quantum Fourier transform (qFT)}

The qFT may be defined by unitary transform on $n$-qubit register

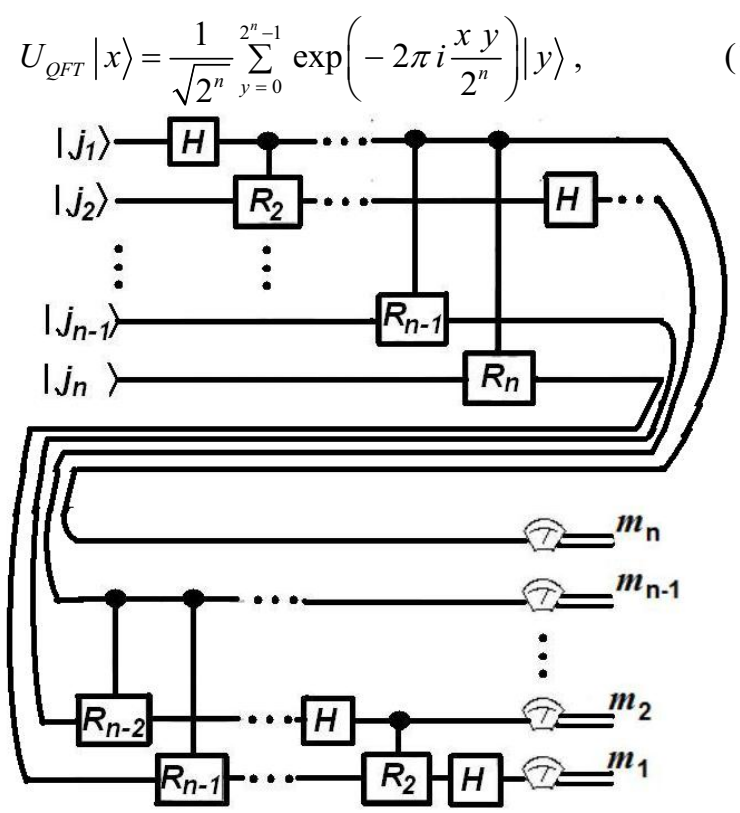

Figure 1. Quantum circuit for exact $\mathrm{qFT}, H \in \mathrm{C}^{(2,2)}$ is the Hadamard gate $\left(H_{i j}=2^{-1 / 2}(-1)^{i j}, i, j \in\{0 ; 1\}\right)$ and $R_{p}$ is the $z$ rotation gate with angle $\omega_{p}=2 \pi / 2^{p}($ $\left.R_{Z}(\omega) \equiv|0\rangle\left\langle 0\left|+e^{i \omega}\right| 1\right\rangle\langle 1|\right)$. Edited picture from [1].

some authors define it with the opposite sign in the exponential above. Straightforward implementation costs $\mathrm{O}\left(n^{2}\right)$ Hadamard gates and controlled phase shifts, efficient $\mathrm{O}(n \log n)$ approximations exist [57].

\subsection{The phase estimation algorithm (PEA)}

The algorithm gives first $m$ bits estimation of the phase $\phi$ by parameterizing given $2^{l} \times 2^{l}$ unitary operator $U$ (matrix, implemented as a quantum logical gate) 
eigenvalue $\lambda=\exp (2 \pi \mathrm{i} \phi)$. The algorithm works with $m$ qubit phase read-out register , $a^{\text {“ }}$ and $l$-qubit eigenvector (wave function) register , $b^{\prime \prime}$ and requires initialization of the latter one to the initial guess $\left|\psi_{0}\right\rangle \in \mathrm{C}^{\wedge}\left(2^{l}\right)$ of the corresponding $U$ eigenvector $|\psi\rangle$.

In applications for bounded states energy estimation (problem of finding Hermitian $H_{\text {ham }}$ Hamiltonian eigenvalues) $U$ is then of the form $U=\exp \left(i \Delta t\left(H_{\text {ham }}-E_{\min } I_{2^{l}}\right)\right)$ and then lower $\left(E_{\text {min }}\right)$ and upper $\left(E_{\max }\right)$ bounds ${ }^{\mathrm{e}}$ for the $H_{\text {ham }}$ eigenvalue $E$ are also needed as an input for the algorithm $\left(E_{\min }<E<E_{\max }\right)$ and $\Delta t$ parameter choice of the form $\Delta t=2 \pi /\left(E_{\max }-E_{\text {min }}\right)$ is used.

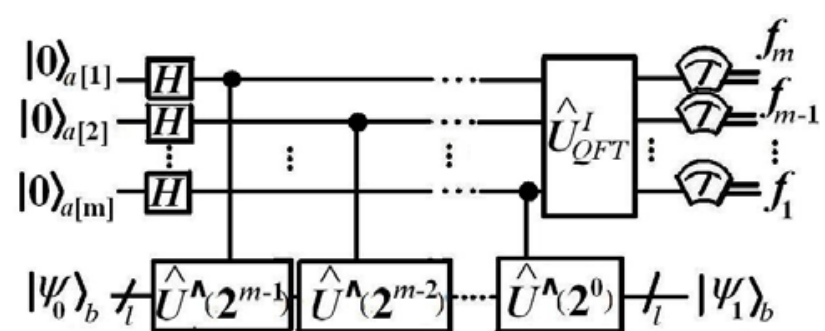

Figure 2. PEA quantum circuit. After Hadamard gates are applied, the equally weighted superposition is created in the register a, the subsequent $\mathrm{m}$ application of the conditioned $U$ gate leads to entangled state (2). Due to the Fourier orthogonality relations it is clear that qFT will amplify amplitude for states of a corresponding to the closest $m$-bit approximations to $\phi$ (see (3)) Also $\left|\psi_{1}\right\rangle$ would be close to the ( $U$ and) $H_{\text {ham }}$ eigenvector (this information is not accessible directly but can be approximately revealed by repeated measurements in different basis sets) $[24,25,1]$.

$$
\begin{aligned}
& \mid \text { core }\rangle=\frac{1}{\sqrt{2^{m}}} \sum_{x=0}^{2^{m}-1}|x\rangle U^{x}\left|\psi_{0}\right\rangle_{b}= \\
& \frac{1}{\sqrt{2^{m}}} \sum_{x=0}^{2^{m}-1}|x\rangle \exp \left(2 \pi i x H^{\prime}\right)\left|\psi_{0}\right\rangle_{b}
\end{aligned}
$$

where $H^{\prime}=\left(H_{\text {ham }}-E_{\min } I_{2^{l}}\right) /\left(E_{\max }-E_{\min }\right)$ is original Hamiltonian rescaled so that its eigenvalue in question lies within $\langle 0 ; 1)$ interval and is therefore represented by the phase $\phi$ of the form

$$
\phi=0 . f_{1} f_{2} \ldots f_{\mathrm{m}}+\delta .2^{-m},
$$

e While the upper bound $E_{\max }$ for the ground state is easy to compute due to the variational theorem (in case we use PEA in connection with Full Configuration Interaction (FCI) computation, we can use the Hartree-Fock energy $E_{H F}$ as the upper-bound), the lower bound $E_{\min }$ is less attainable, the Frobenius Theorem or similar algebraic bounds may be used, unfortunately this would mean $\mathrm{O}\left(\left(2^{l}\right)^{\wedge} 2\right)$ costly classical precalculation $[26,27]$. It would be interesting to note some works dedicated to lower bounds, e.g. [28-30]. For lower estimate, the $E_{H F}-$ $\alpha\left(E_{H F-} E_{M B P T}\right)$ for some $\alpha>1$ (MBPT stands for the Many Body Perturbation Theory) might be used [61].
The algorithm is based on quantum circuit above and (usually very tight) lower bound to the success probability $p_{m}$ defined as the sum of probabilities of the two closest approximations to the correct phase $\phi$ equals

$$
p_{m}(\delta) \geq\left|\left\langle\psi_{0} \mid \psi\right\rangle\right|^{2} \cdot S_{m}(\delta)
$$

where $S_{m}(\delta)$ range is $\left\langle 8 / \pi^{2} ; 1\right\rangle \dot{=}=\langle 0.81 ; 1\rangle$, exact form can be found e.g. in [3]. Therefore for algorithm to be useful, the sufficient condition on eigenvector initial guess is that its overlap with the eigenvector exact within given finite computational basis is to be $\left|\left\langle\psi_{0} \mid \psi\right\rangle\right|^{2} \geq 0.62$

\subsection{Iterative PEA (IPEA)}

Through the idea of „measurement conditioned operations“ " [31] we can „decouple“ individual $\phi$ 's bits estimation measurement and lower the qubit requirements on read-out register from $m$ qubits to 1 qubit at the cost of repeated operation of quantum circuit in Fig.? in the next paragraph where angle $\omega_{k}$ parameterizing $z$-rotation gate depends on previous bit measurements (starting from the least significant bit $\phi_{m}$ ).

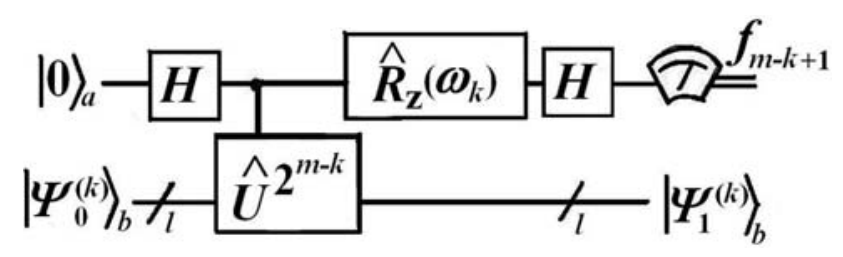

Figure 3: Iterative PEA quantum circuit. The algorithm starts for $k=1$, the angle wk depends on the previously measured bits so $\omega_{k}=2 \pi \cdot 0.0 f_{\mathrm{m}-\mathrm{k}+2} f_{\mathrm{m}-\mathrm{k}+3 \ldots} . f_{\mathrm{m}}$. In case we maintain the $b$ register for all iterations $\left(\left|\psi_{0}^{(k+1)}\right\rangle_{b}=\left|\psi_{1}^{(k)}\right\rangle_{b}\right)$ the term "IPEA A" will be used. Another possibility (favourable in case the coherence time is too short) is to prepare the same state at the beginning of each iteration $\left(\left|\psi_{0}^{(k)}\right\rangle_{b}=\left|\psi_{0}\right\rangle_{b}\right)$ in this case the term "IPEA B" will be used. Unlike IPEA A, the IPEA B is not fully equivalent to original PEA and the success probability formula (4) doesn't hold for this case. Instead, lower and upper estimates $\left|\left\langle\psi_{0} \mid \psi\right\rangle\right|^{2 m-2} \bigcirc p_{m} / S_{m}(\delta) \bigcirc\left|\left\langle\psi_{0} \mid \psi\right\rangle\right|^{m-1}$ can be derived [32]. This would lead to practical uselessness since even for a high overlap, the $p_{m}$ would decrease with $\mathrm{m}$ quickly under 0.5 . However, if each iteration in IPEA B is $r$-times repeated and bits are decided by the majority vote, for $r>3$, the IPEA B pm usually surpass the IPEA A value. IPEA B is likely to work even when overlap is lower than 0.62 or even 0.5 as long as the eigenvector $|\psi\rangle$ keeps to have highest overlap with the initial eigenvector guess from the $2^{l}$-tuple set of all $H_{\text {ham }}$ eigenvectors.

\subsection{Abrams-Lloyd algorithm}

The idea is to simply exploit the (I)PEA for physical Hamiltonians. First introduced by Abrams and Lloyd into quantum chemistry in the second quantized formulation [12-16].

Since the $U$ operator is usually exponential of the sum of non-commuting simple operators, the Trotter-Suzuki 
formulae of various orders [33] are used for its implementation (the exponentials of the simple operators are easily decomposed into single qubit gates and CNOTs). The total gate count for (I)PEA algorithm scales as $\mathrm{O}(-X \cdot \ln (\Delta E \delta E) / \delta E)$, where $X$ is the asymptotical cost for $U$ operator implementation (usually in form $\mathrm{O}\left(N^{a}\right.$ $\left.\log ^{b} N\right)$, where $N$ is the size of system studied, or size of the one-particle basis set and $a$ and $b$ are small and positive), $\Delta E=E_{\max }-E_{\min }$ and $\delta E=2^{-\mathrm{m}} \Delta E$ is the precision in energy (should be chosen as slightly higher than expected magnitude of error due to the finiteness of basis used).

The particular mapping between algebra of operators acting on the Hilbert space of physical system we are studying and Hilbert space of qubits and corresponding mapping of Hilbert spaces is matter of the next paragraphs.

\subsubsection{First quantized formulation}

Let us consider a simple fully non-relativistic Hamiltonian of interacting $A$-body system (without the center-of-mass (CM) movement separation, interactions are pair-wise, momentum and spin independent and local in position basis). The first quantized quantum algorithm was first introduced by Weisner and Zalka [34,35].

$$
\hat{H}=\sum_{j=1}^{A} \frac{\hat{p}_{j}^{2}}{2 m_{j}}+\sum_{j, k} \hat{V}_{j k}\left(\vec{r}_{j}, \vec{r}_{k}\right),
$$

The wave function of this system is stored ,on (cartesian) grid" in $3 A \cdot(b+z)$-qubit register, where $B=2^{b}$ is number of points of the grid per each cartesian coordinate (chosen as some power of two) and $z$ corresponds to spin variable and is usually a small number compared to $b$ (for spin-1/2 fermions $z=1$ ). It can be shown that when the initial guess wave-function $\left|\psi_{0}\right\rangle$ is properly (anti)symmetrized with respect to variables of indistinguishable particles of given statistics (Fermi, Bose), the (anti)symmetry is kept within the scope of quantum diagonalization algorithm [36]. The partitioning of $\hat{H}$ in the exponent in $\hat{U}$ for Trotterization is then

$$
\begin{aligned}
& \hat{U}=\exp (i \Delta t(\hat{T}+\hat{V}))= \\
& \lim _{m \rightarrow \infty}\left(\exp \left(i \frac{\Delta t}{m} \hat{T}\right) \exp \left(i \frac{\Delta t}{m} \hat{V}\right)\right)^{m}
\end{aligned}
$$

or for second order Trotter-Suzuki [33] formula

$$
\begin{aligned}
& \hat{U}=\exp (i \Delta t(\hat{T}+\hat{V}))= \\
& \lim _{m \rightarrow \infty}\left(\exp \left(i \frac{\Delta t}{2 m} \hat{V}\right) \exp \left(i \frac{\Delta t}{m} \hat{T}\right) \exp \left(i \frac{\Delta t}{2 m} \hat{V}\right)\right)^{m}
\end{aligned}
$$

where $\hat{T}$ stands for the sum of kinetic energy operators and $\hat{V}$ for the sum of potential energy operators, the latter being dependent on position only, the former on momentum only.

The $\hat{V}$ and therefore $\exp (\mathrm{i}(\Delta t / m) \hat{V})$ are diagonal within position basis and corresponds to $6 \mathrm{~b}$ qubit gate acting on wave-function register. It can be shown, that best up to now known algorithms use at most $\mathrm{O}(b)$ oneand two-qubit gates for its decomposition.

The $\hat{T}$ and therefore $\exp (\mathrm{i}(\Delta t / m) \hat{T})$ are diagonal within momentum basis and are similar to diagonal operators $\hat{T}_{j}^{(p)}$ (j indicates coordinate not particle) and $\exp \left(\mathrm{i}(\Delta t / m) \quad \hat{T}_{j}^{(p)}\right)$ respectively via $\mathrm{qFT} \quad\left(\hat{U}_{Q F T, j}\right.$ in formula (8) below)

$$
\exp \left(i \frac{\Delta t}{m} \hat{T}\right)=\prod_{j=1}^{3 A} \hat{U}_{Q F T, j}^{+} \exp \left(i \frac{\Delta t}{m} \hat{T}_{j}^{(p)}\right) \hat{U}_{Q F T, j},
$$

The gate costs are $\mathrm{O}(b)$ for each exponential and either $\mathrm{O}\left(b^{2}\right)$ (for „exact“" qFT) or $\mathrm{O}(b \log b$ ) (for approximate qFT) on the right side of (8).

Therefore the total gate cost for implementing one time-step of Trotter-Suzuki formula [33] (simple product of the two exponentials in (6) or three exponentials in (7)) is either $\mathrm{O}\left(A^{2} b\right)+\mathrm{O}\left(A b^{2}\right)$ or $\mathrm{O}\left(A^{2} b\right)+\mathrm{O}(A b \log b)$ depending on our qFT implementation. The cost of the algorithm also depends on the scaling of the time-steps number $m$ needed in (6) with $A$ and $b$ for a constant error in eigenvalue due to Trotter formula error. Rough examination based on the (15) formula from [37] gives $m$ $=\mathrm{O}\left(A^{3 / 2}\right)$ (at least for a large $b, m$ would not depend on $b$ ) implying the total gate cost of algorithm to scale as $\mathrm{O}\left(A^{3.5}\right.$ $b)+\mathrm{O}\left(A^{2.5} b^{2}\right)$ or $\mathrm{O}\left(A^{3.5} b\right)+\mathrm{O}\left(\mathrm{A}^{2.5} b \log b\right)$ depending on our $\mathrm{qFT}$ implementation. This result shows quantum algorithm to be efficient when compared to classical FCI exponential scaling with $A$. Even for the lowest values of $A$, the quantum algorithm poses an advantage over its classical counter-parts due to the cost scaling with respect to $b=\log _{2} B$ (diagonalization of a sparse matrix of dimension $\mathrm{O}(B)$ has $\mathrm{O}\left(B^{2}\right)$ time-cost which is exponentially larger than at most quadratic scaling in log $B)$. The cartesian grid is assumed here to be equidistant running from $-B \cdot d r / 2$ to $+B \cdot d r / 2$ in each coordinate axis with elementary step equal to $d r$. The eventual nonequidistant grid computation probably would not need large overhead. First quantization is also addressed in the works [38] and [39].

The separation of CM movement might be done by coordinate system choice explicitly (e.g. Jacobi coordinates, typical for small $A$ ) or by subtracting the total kinetic energy of the system. The subtraction leads to masses $m_{j}$ being replaced by reduced masses $\mu_{j}^{-1}=m_{j}^{-1}$ - $M^{-1}\left(M=\Sigma_{j} m_{j}\right)$ and $\hat{V}_{j k}$ now containing term ($1 / M)\left(\hat{\vec{p}}_{j} \cdot \hat{\vec{p}}_{k}\right)$. The latter should be for the purpose of Trotter formula [33], however contained in the $\hat{T}^{\prime}$ operator (the prime distinguish the operator from the case where CM movement is not separated) since it commutes with other momentum-dependent operators. Now

$$
\begin{aligned}
& \exp \left(i \frac{\Delta t}{m} \hat{T}^{\prime}\right)=\exp \left(i \frac{\Delta t}{m} \hat{T}\right) \\
& \prod_{k<j=1}^{3 A} \hat{U}_{Q F T, k}^{+} \hat{U}_{Q F T, j}^{+} \exp \left(-i \frac{\Delta t}{m M} p_{j} p_{k}\right) \hat{U}_{Q F T, j} \hat{U}_{Q F T, k}
\end{aligned}
$$


meaning $\mathrm{O}\left(A^{2} b^{2}\right)$ gate cost for this part of Trotter-Suzuki expansion [33] and also for one Trotter time-step. Rough estimate for $m$ in Trotter formula gives now $m=\mathrm{O}\left(A^{2}\right)$ implying the total gate cost $\mathrm{O}\left(A^{4} b^{2}\right)$. Similar gate counts would be expected for Hamiltonian (5) in form of sum of Dirac-like Hamiltonians (Dirac-Coulomb or DiracCoulomb-Breit Hamiltonian). In case of three-body forces $\hat{V}_{j k n}\left(\vec{r}_{j}, \vec{r}_{k}, \vec{r}_{n}\right)$, or more generally for the Hamiltonian containing $N$-body forces $\hat{V}_{j_{1} j_{2} \cdots j_{N}}\left(\vec{r}_{j_{1}}, \vec{r}_{j_{2}}, \cdots, \vec{r}_{j_{N}}\right)$ the gate count would be increased up to $\mathrm{O}\left(A^{N} b\right)+\mathrm{O}\left(A^{2} b^{2}\right)$ for a single Trotter time-step and $m=\mathrm{O}\left(A^{N}\right)$, therefore the total cost would be $\mathrm{O}\left(A^{2 N} b\right)+\mathrm{O}\left(A^{N+2} b^{2}\right)$.

However, the nuclear structure theory works with momentum dependent interaction potential, like HamadaJohnson [40] or Argonne [41]. In this case, since the potential depends solely on the angular-momentum (but non-linearly) spherical coordinates are natural and we can think about discretizing only the radial coordinate, so the single-particle wave-function has a form

$$
|\psi\rangle=\sum_{j, m_{j}, l, s, \tau} R_{j, m_{j}, l, s, \tau}(r)\left|j, m_{j}, l, s\right\rangle|\tau\rangle,
$$

then the wave-function register would be divided into few-qubits parts storing angular momentum information, isospin and similar information and $b$-qubit radial part for $R(r)$ stored for discrete values of $r$ from $d r$ to $2^{b} d r$. While implementation is straightforward for the two-nucleon problem, in case of three- and more nucleon problems, further formulae would need to be derived (e.g. multipole expansion of $r$-dependent part of potential into radial and angular coordinates of interacting nucleons).

For this case, the non-relativistic kinetic energy operator should be written in spherical coordinates as

$$
\hat{T}=\frac{\hat{p}_{r}^{2}}{2 \mu}+\frac{\hat{L}^{2}}{2 \mu r^{2}},
$$

where $\hat{p}_{r}^{2}=-r^{-1} \partial_{r}^{2} r$ (note: $\partial_{r}$ is the derivative with respect to $r$ operator). From [42] we can conclude that similar trick as with linear momentum could be done with the radial momentum operator only with quantum discrete sine transformation used instead of the qFT with the same gate cost of $\mathrm{O}\left(b^{2}\right)$ for the quantum sine transform [43].

In [38] rigorous description of different algorithm using $3 A$. $(B+\mathrm{z})$-qubit wave-function register with easier implementation, but with scaling at least $\mathrm{O}(B \log B)$ with the respect to grid point number for a single Trotter step is presented. However, the [38] version allows simple adaptation of the algorithm to irregular space-sampling (i.e. no rectangular grid at al).

\subsubsection{Second quantized formulation}

Let us consider the simplest second quantized Hamiltonian

$$
\hat{H}=\sum_{j, k}^{N, N} h_{j k} a_{j}^{+} a_{k}+\sum_{j<k, l<m}^{N, N, N, N} V_{j k l m} a_{j}^{+} a_{k}^{+} a_{m} a_{l},
$$

where $h_{j k}=h_{k j} *$ and $V_{j k l m}=V_{k j m l}=V_{m l j k} *$ are complex numbers and $a_{j}^{+}$(or $a_{j}$ ) are creation (or annihilation) operators for the single particle basis states fulfilling the (anti-)commutation relations for bosons (for the bosonic case, they will be marked as $b_{j}^{+}$) (14) (fermions (13)).

$$
\begin{aligned}
& \left\{a_{j}^{+}, a_{k}\right\}=\delta_{j k}, \quad\left\{a_{j}^{+}, a_{k}^{+}\right\}=\left\{a_{j}, a_{k}\right\}=0, \\
& {\left[b_{j}^{+}, b_{k}\right]=\delta_{j k}, \quad\left[b_{j}^{+}, b_{k}^{+}\right]=\left[b_{j}, b_{k}\right]=0,}
\end{aligned}
$$

The Hamiltonian can be further generalized by adding three- $\left(\left(1 /(3 !)^{2}\right) \sum_{j k l m p q} W_{j k l m p q} a_{j}^{+} a_{k}^{+} a_{l}^{+} a_{q} a_{p} a_{m}\right)$, or more particle terms and/or by adding another kind of particles with corresponding different set of creation and annihilation operators (including the interaction terms between those two kinds of particles).

\subsubsection{The direct mapping}

For the fermionic case, we can thing about either compact or direct mapping of the Hilbert space of interacting system described by (12) onto quantum register and corresponding mapping of algebras preserving the anticommutation relations (13). In the case of direct mapping, the Jordan-Wigner transformation (JWT) [44] where qubits store occupation numbers for spin-orbitals can be used - in this case, the creation operator is realized by $\mathrm{O}(N)$ sequence of Pauli matrices acting on the quantum register $(15)^{\mathrm{f}}$.

$$
a_{p}^{+} \stackrel{J W T}{=}(-1)^{p-1} Z_{1} Z_{2} \cdots Z_{p-1} \sigma_{p,+} .
$$

Later, a more effective, Bravyi-Kitaev transformation (BKT) (16) was developed [45-46] - in this case qubits store rather specific partial sums of occupation numbers and creation (and annihilation) operators are realized by just $\mathrm{O}(\log N)$ sequence of Pauli matrices.

$$
a_{p}^{+} \stackrel{B K T}{=} X_{U(p)} \sigma_{j,+} Z_{R(p)},
$$

here, $U(p)$ and $R(p)$ are sets with $\mathrm{O}(\log N)$ elements - for closer description see [45-46]. For the case of distinguishable particles, $a_{p}^{+}$is represented by $\sigma_{k}^{+}$alone. For the bosonic particles, transformations preserving the commutation relations (14) (respectively their projection onto finite dimensional Fock subspace - note: we can store only states with maximum $V_{p}$ bosonic particles in the $p$-th spin-orbital when $p$ is finite) have been developed too - Somma et al [47] presents simple and powerful transformation with gate cost $\mathrm{O}\left(V^{4} N^{4}\right)$ for the two-particle term in (12) where $V=\max _{\mathrm{p}} V_{p}$, the qubit cost for representing $p$-th spin-orbital occupation number is, however $V_{p}+1$. Different transformation for bosonic particles, using only $\left\lceil\log _{2}\left(V_{p}+1\right)\right\rceil$ qubits with $\mathrm{O}\left(U^{8} N^{4}\right)$

\footnotetext{
${ }^{\mathrm{f}} Z_{k}, X k, \sigma_{k,+}$ are $k$-th qubit Pauli $\sigma_{z}, \sigma_{x}, \sigma_{+}=(1 / 2)\left(\sigma_{x}+i \sigma_{y}\right)$.
} 
gate cost for the two-particle term in (12) will be presented in article [48].

Through the Trotter-Suzuki formula [33], the evolution operator $U$ for (12) Hamiltonian is decomposed into the products of exponentials of $\mathrm{i}(\Delta \mathrm{t} / \mathrm{m})$ multiples of individual terms in (12) and each of them is realized by $\mathrm{O}\left(\log ^{4} N\right)$ gates. This means $\mathrm{O}\left(N^{4} \log ^{4} N\right)$ gates for applying $U$ in total (I will address the three-particle force generalization briefly at the end of the next paragraph). In the case of local basis (and $N$ denoting size of the system when using always the same ratio of one-particle basis set dimension to number of centers/particles) we can even thing about just $\mathrm{O}\left(N^{2} \log ^{4} N\right)$ cost since only $\mathrm{O}\left(N^{2}\right)$ terms will be then non-negligible (or even $\mathrm{O}(N)$ in a truly asymptotic region) [49]. Although the scalings cited in the next paragraph came from electronic structure theory, they provide probably rather good estimation and worst scenario bounds [50] for the nuclear structure theory as well.

The trotter number $m$ can be estimated to scale at worst as $m=\mathrm{O}\left(N^{6}\right)$ [50], but fortunately realistic simulations done on electronic structure problems show that the scaling is rather $m=\mathrm{O}\left(N^{4}\right)$ [50], Poulin et al [51] presented scaling $m=\mathrm{O}\left(N^{1.5-2.5}\right)$ for a different, more realistic set of molecules. By simple multiplication of $\mathrm{m}$ with the gate counts for implementing $U$ gate from previous paragraph, the total gate (and therefore time) cost of algorithm will range from $\mathrm{O}\left(N^{10} \log ^{4} N\right)$ through $\mathrm{O}\left(N^{8} \log ^{4} N\right)$ [50], $\mathrm{O}\left(N^{5.5-6.5} \log ^{4} N\right)$ [51] to $\mathrm{O}\left(N^{3.5-4.5} \log ^{4}\right.$ $N)[51,49]$. In the case of $q$-particle $(q>2)$ force terms in Hamiltonian we have to add 2(q-2) (or $q$-2 with local one-particle basis set, [49]) to the power of $N$ in the computational costs.

The most straightforward choice for the one-particle basis set are the ground state Hartree-Fock spin-orbitals (Mean-field solutions), however any orthonormal basis set complete enough for appropriate precise description could be used. The Hartree-Fock (HF) many-body wavefunction solution (Slater determinant for the ground state) is also the most straightforward candidate for the initial eigenvector guess $\left|\psi_{0}\right\rangle$, in case it will show to have too small overlap for success probability over 0.5 , one can either use some state from classically carried out post-HF method (usually only few Slater determinant are necessary) or use quantum algorithms - Adiabatic (quantum) State Preparation (ASP) [13, 52] or the quantum cooling [53] methods for the $\left|\psi_{0}\right\rangle$ initialization.

It would be interesting to do similar simulations for nuclear structure problems in order to evaluate the success probabilities for HF initial guess and to derive how the Trotter number scales with the one-particle basis size and number of nucleons of the system. Work on this matter is in progress (in the collaboration with P. Veselý from Nuclear Physics Institute of the ASCR).

In case of the nucleon-nucleon mechanical approach one can either use isospin formalism or to think about neutrons and protons as two different kind of particles having their respective sets of spin-orbitals and corresponding creation and annihilation operators (let us say $a_{j}^{+}$'s and $a_{J}^{+}$s, where $j$ and $J$ are from two nonoverlapping sets (so the (12) form holds, just summation indices change their meaning) and $\left[a_{j}^{+}, a_{K}\right]=\left[a_{j}^{+}, a_{K}{ }^{+}\right]=0$ - operators on proton and neutron space commute with each other).

\subsubsection{The compact mapping}

While the Hamiltonian in the form (12) conservate number of particles, in direct mapping the quantum register still can superposition of states from Fock spaces corresponding to different particle numbers (from the physical vacuum up to system with all (including the virtual) spin-orbitals filled) - this seems we are wasting with, at least, the qubit resources (however the bottleneck for quantum computers and quantum computation will sooner or later show to be rather the number of gates and computational time respectively (which should be always much lesser than the decoherence time)). Fortunately, algorithms storing only $A$-particle subspace of the Fock space onto $\mathrm{O}\left(\log _{2} \mathrm{C}(N, A)\right)$ qubit register $(N$ is the number of basis spin-orbitals, $A$ number of particles (= filled spinorbitals) and $C(N, A)$ the combination number) have been developed too and give rise to $U$ implementation costs of $\mathrm{O}\left((N-A)^{4 / 3} \log \log (N-A)\right)$ in general case [20] and $\mathrm{O}((N-$ $\left.A)(\log (N-A))^{7 / 4}\right)$ using black-box algorithms [20, 54, 55]. Unfortunately little to no studies were done for the total one- and two-qubit gate cost for this case.

\subsubsection{Quantum field theory}

While in the case of first quantized Abrams-Llyod qA we would think about the Lattice QCD application, the both lattice QCD and non-lattice QCD computation within the second quantized variants are also of possible further interest. Since the direct mapping, the compact mapping and their eventual hybrids might be used also for efficient simulation of quantum field theory Hamiltonians nonconserving number of particles.

\subsection{Alternative algorithms}

From the plethora of alternative algorithms for Hamiltonian eigenvalue estimation on quantum computers, the Quantum expectation estimation (QEE) should be mentioned [56], it is based on the second quantized Hamiltonian (12) with (JWT) or (BKT) this results in Hamiltonian represented as a linear combination of strings of Pauli matrices - each of the strings are applied to quantum register representing the wave-function and the register is $\mathrm{O}\left(|h|^{2} / p^{2}\right)$ measured to obtain contribution to energy with the precision $p$. After that, parameters of state preparation are changed in order to lower energy estimated. After minimum with respect to the preparation parameters is achieved, we can conclude we had variationally found ground state energy [56]. The gate cost for this algorithm is $\mathrm{O}\left(N^{2 q}\left|h_{\max }\right|^{2} / p^{2}\right)$, where $q=2$ for Hamiltonian with at most pair-wise interaction, $q=3$ for Hamiltonian with three-body forces, etc. and $\left|h_{\max }\right|$ is the largest from $h_{j k}, V_{j k l m}$ parameters absolute value. The interesting difference with respect to the (I)PEA is maximal coherence time needed, which here doesn't scale linearly with number of gates used, but is $\mathrm{O}(N)$ for JWT or even $\mathrm{O}(\log N)$ for BKT. The 
drawback is necessity to proceed probably time costly minimizing procedure.

For finding excited state [56] suggests construction $\Delta^{2} H=(H-E)^{2}$ operator ( $E$ is estimate of eigenvalue in question), which increase the computational time to $\mathrm{O}\left(N^{4 q}\left|h_{\max }\right|^{4} / p^{2}\right)$. Similar approach might be used for potential optimization (with respect to parameter set $\beta$ ) $<H(\beta)>$ is first minimized with respect to the state preparation and for this states $\left\langle\Delta^{2} H(\beta)>=<(H(\beta)-E)^{2}>\right.$ is minimized with respect to $\beta$. The computational acceleration is due to inutility to store exponentially large wave-function for each computation and would be exponential. By this process, nucleon-nucleon potentials, three- and four- body forces can be fitted to bonding energies of several nuclei.

\section{Deuterium and Tritium simulation}

From the scaling derived for second quantized approach it is obvious that this framework will favourite quantum computers over the classical ones rather for much higher nucleonic number $A$ (like $A>10$ ). For the simplest systems in question, first quantized formulation is the only option providing quantum algorithmic speed-up. Unfortunately, only plan of further simulation will be presented - I would start with Jacobi coordinates, isospin formalism and in case of deuterium, the wave function would be written as

$$
|\psi\rangle=|S\rangle+|D\rangle
$$

Where (18-22)

$$
\begin{aligned}
& \langle r=r \mid S\rangle= \\
& \frac{R_{S}(r)}{r}\left|J=1, m_{J}=0, l=0, s=1\right\rangle_{L, \text { spin }} \otimes|T=0\rangle_{\text {isospin }} \\
& \langle r=r \mid D\rangle= \\
& \frac{R_{D}(r)}{r}\left|J=1, m_{J}=0, l=2, s=1\right\rangle_{L, \text { spin }} \otimes|T=0\rangle_{\text {isospin }} \\
& |T=0\rangle_{\text {isospin }}=\frac{1}{\sqrt{2}}(|n p\rangle-|p n\rangle), \\
& \left\langle\theta, \varphi \mid J=1, m_{J}=0, l=0, s=1\right\rangle_{L, \text { spin }} \\
& \quad=Y_{0,0}(\theta, \varphi) \frac{1}{\sqrt{2}}(|\uparrow \downarrow\rangle+|\downarrow \uparrow\rangle) \\
& \left\langle\theta, \varphi \mid J=1, m_{J}=0, l=2, s=1\right\rangle_{L, \text { spin }}= \\
& \sqrt{\frac{3}{10}}\left(Y_{2,-1}(\theta, \varphi)|\uparrow \uparrow\rangle+Y_{2,+1}(\theta, \varphi)|\downarrow \downarrow\rangle\right) \\
& \quad-\sqrt{\frac{2}{5}} Y_{2,0}(\theta, \varphi) \cdot \frac{1}{\sqrt{2}}(|\uparrow \downarrow\rangle+|\downarrow \uparrow\rangle)
\end{aligned}
$$

for the both Hamada-Johnson and Argonne NNpotentials, the matrix elements between angular, spin and isospin parts of the wave function of the following operators would be of high importance $-\hat{\vec{\tau}}_{1} \cdot \hat{\vec{\tau}}_{2}, \hat{\vec{\sigma}}_{1} \cdot \hat{\vec{\sigma}}_{2}$, $\hat{S}_{12}, \quad \hat{L}_{12}, \quad(\hat{\vec{L}} \cdot \hat{\vec{s}}) \quad$ and $\quad(\hat{\vec{L}} \cdot \hat{\vec{s}})^{2} \quad$ (please see the Supplementary Information [59]).

The radial part of the non-relativistic kinetic energy operator action on $|\psi\rangle$ is discussed in detail in "First quantized formulation and its future for Computational Nuclear Physics" section. The wave-function quantum register would consist of $b$ qubits (starting with $b=4$ and hopefully extended up to 10 or more) for storing radial part $\left(R_{S}(r)\right.$ or $\left.R_{D}(r)\right)$ of the wave function and one qubit for distinguishing $S(|0\rangle)$ and $D(|1\rangle)$ components.

The wave function register should be initialized into the sampled wave-function corresponding to the variational solution of the stationary Schroedinger equation corresponding to the Hamada-Johnson or Argonne NN-potentials (with the non-relativistic form of the kinetic energy term $\hat{p}^{2} /(2 \mu)$, where $\mu$ is the reduced mass) in the form (23)

$$
R_{a}(r)=r^{l+1}\left(\left(r / r_{0}\right)^{\sigma_{a}}-1\right)^{\varepsilon_{a}} \sum_{k=0}^{P} c_{a, k} r^{k} \exp \left(-\eta_{a}\left(r-r_{C}\right)^{\alpha_{a}}\right)
$$

where $a \in\{S, D\}, l=0$ for $a=S$ and $l=2$ for $a=D, P$ represents size of the basis $(P=0$ for the first simulations), $\sigma_{a}, \varepsilon_{a}>0$ are variational parameters (this part $\left(\left(\left(r / r_{0}\right)^{\sigma_{a}}-1\right)^{\varepsilon_{a}}\right)$ of the radial wave function assures the hard-core behaviour and is applied only for HamadaJohnson potential - for $r>r_{0}$, for $r \leq r_{0}$ we put $R_{a}(\mathrm{r})=0$, eventually leading to reinitialization of the corresponding wave-function register part every $p$-th Trotter step for some reasonable positive integer $p$ ) as well as complex numbers $c_{a, k} \in C$, and parameters of the exponential part $-r_{C}, \eta_{a}$ and $\alpha_{a}>0$. For the sake of simplicity $\alpha_{a}$ should be either 1 or 2 . The former would be preferable since it corresponds to the correct asymptotic behaviour in infinity (similar to the hydrogen atom, since the dominant part of potential in this region scales as $1 / r$ ). The very general formula (23) may be, however, simplified for the preliminary simulations by setting $P=0, \alpha_{a}=\sigma_{a}=\varepsilon_{a}=1$ (or $\varepsilon_{a}=0$ and therefore omitting this term in case of the soft-core Argonne potential) and $\eta_{S}=\eta_{D}=\eta$ would be the only parameter for classical precomputation via variational minimization of energy expectation value.

There is another way of dealing with the hard-core behaviour of Hamada-Johnson potential or with integral divergence (when radial part (23) is used), in case of the soft-core Agronne potential is introducing different form of the radial wave-function containing inverse powers of $r$ inside the exponential, i.e.

$$
R_{a}(r)=r^{l+1} \sum_{k=-Q}^{P} c_{a, k} r^{k} \exp \left(-\eta_{a,-b} r^{-b}-\eta_{a, c} r^{c}\right)
$$

where $Q$ is a small positive integer, $b, \mathrm{c} \in\{1 ; 2\}$ (all four combination possible) and $\eta_{a,-b}, \eta_{a, c}>0$. This kind of wave-function should assure convergence of all integrals over negative power of $r$ due to the $-\eta_{a,-b} r^{-b}$ term in the exponential. Rough inspection led me to think that all 
integrals in this case are also analytical and in the case of $b=c=1$ could be expressed in the form of BesselK functions, in the $b=2, c=1$ case in the form of Gamma and HypergeometricPFQ functions (according to the Mathematica [58] terminology). In case of HamadaJohnson potential and wave-function of the form (24) the hard-core may be replaced by replacing parts of potential of the $\sim r^{-a}$ kind (only for $r<r_{0}$ ) into linear combination of two (or eventually more) functions $\left(A r^{-b}+B \mathrm{r}^{-c}\right)$ fulfilling equality of function values and first derivatives with respect to $r$ in the $r=r_{0}$ point, $b$ and c should be then chosen to be large enough to emulate the hard-core behaviour. Due to the $-\eta_{a, b} r^{-b}$ term in the exponential in (24) this would lead to analytical and finite expression for all integrals. And for the quantum simulation this would mean no need for reinitializing of the wave-function register in order to assure that its $r<r_{0}$ part remains zero (in this case it would be just sufficiently small).

I must apologize to the readers that I am stopping here, but computational details and progress in the work on the simulation of quantum algorithm for deuterium energy estimation in the first quantization will be placed on the web [59].

After the simulation (with $m$ fixed to $m=17$ and properly chosen $E_{\max }$ and $E_{\min }$ (preferably based on lower bounds from $[28,29,30])$ ) I would like to address this questions:

1. How the correct energy and its $m$ bit estimations scales with the number of grid points $2^{b}$ and the $d r$ parameter?

2. What was the IPEA A and IPEA B success probabilities $p_{m}$ for several possible initialization of wave-function register $\left|\psi_{0}\right\rangle$ and values of $b$ and how is this value correlated with the overlap $\left|\left\langle\psi_{0} \mid \psi\right\rangle\right|^{2}$ ? For this task, oracle-like application of controlled- $U$ gate would be sufficient.

3. What was the necessary value of the Trotter number and how it depends on the grid point parameter $b$ ? How many elementary gates would be needed on ideal quantum computer for the computation?

Then, similar simulation I plan with tritium ground state energy calculation.

\section{Conclusions}

This work aim is to approach the quantum algorithm design to the Computational Nuclear Physics community, to present gate count estimations for first quantized formulation of the Abrams-Lloyd algorithm for nuclear structure problems (in the "First quantized formulation and its future for Computational Nuclear Physics" section). The preparative steps for the simulations of quantum algorithms for bounded state energy calculation for the smallest two nuclei were presented in the previous

$\mathrm{g}$ The maximal largest radial coordinate value in the computation is then $2^{b} d r$, the smallest $d r$. This question is rather technical and has not much to do with quantum algorithms, however, it is important for the eigenvalue problem in question. section (Deuterium and Tritium as first systems for the simulation).

\section{Future prospects}

Author, in the collaboration with Veselý from Nuclear Physics Institute of the ASCR plans to proceed simulations of second-quantized Abrams-Lloyd algorithm for ${ }^{4} \mathrm{He},{ }^{16} \mathrm{O}$ and ${ }^{40} \mathrm{Ca}$ nuclei and question the success probabilities and Trotter number scaling.

\section{Acknowledgements}

I would like to thank the Department of Nuclear Chemistry for support, my supervisor at Chemical Physics, Jiří Pittner, for introduction into Quantum Information Theory, Petr Veselý for many advices and the Organizing Committee of the TESNAT 2015 workshop for hospitality and the opportunity to present my work.

\section{References}

1. M.A. Nielsen, I.L. Chuang, Quantum Computation and Quantum Information (Cambridge University Press), (2000).

2. J. Gruska: Quantum Computing, Advanced Topics in Computer Science Series, The McGraw-Hill Companies, ISBN 007709503 0.F (1999).

3. M. Dobšíček: Quantum computing, phase estimation and applications, Ph.D. thesis, Czech Technical University in Prague, Faculty of Electrical Engineering, Department of Computer Science and Engineering, (2008).

4. S.J. Aaronson: Limits on Efficient Computation in the Physical World, Dissertation, University of California, Berkeley, (2004).

5. R.P. Feynmann, Int. J. Theor. Phys., 21, 6/7, (1982).

6. D. Deutsch, R. Jozsa, Proc. R. Soc. London A 439: 553-558 (1992).

7. R. Cleve, A. Ekert, C. Macchiavello, M. Mosca, Proc. R. Soc. London A 454: 339-354 (1998).

8. C.H. Bennett, E. Bernstein, G. Brassard, U. Vazirani, SIAM J. Compt. 26(5): 1510-1523 (1997).

9. P.W. Shor, Proc. 35th Ann. IEEE Symp. Found. p. 124 (1994).

10. http://phys.org/news/2014-09-quantumteleportation.html

11. F. Bussières, et al., Nature Photon., DOI: 10.1038/nphoton.2014.215.

12. D.S. Abrams, S. Lloyd, Phys.Rev.Lett. 83, 5162 (1999).

13. A. Aspuru-Guzik, et al., Science 309, 1704 (2005).

14. H. Wang, et al., Phys. Chem. Chem. Phys. 10, 53885393 (2008).

15. J.D. Whitfield, J. Biamonte, A. Aspuru-Guzik, Mol. Phys. 109, 735 (2011).

16. L. Veis, J. Pittner, J. Chem. Phys. 133, 194106 (2010).

17. B.P. Lanyon, et al., Nat. Chem. 2, 106 (2010). 
18. L. Veis, J. Višňák, T. Fleig, S. Knecht, T. Saue, L. Visscher, J. Pittner, Phys. Rev. A 85, 030304(R), 2012.

19. Ch.-L. Wu, CJP, 34, No. 3-II (1996).

20. B. Toloui, P.J. Love, J. Chem. Phys. arXiv:1312.2579v2, (2014).

21. K.G. Dyall, K. Faegri, Introduction to Relativistic Quantum Chemistry (Oxford University Press, New York, 2007).

22. W.P. Shor, Phys. Rev. A 52, R2493(R), 1995.

23. D. Gottesman, Quantum Information Science and Its Contributions to Mathematics, Proc. Sympos. Appl. Math. 68, pp. 13-58 (Amer. Math. Soc., Providence, Rhode Island, 2010), arXiv:0904.2557.

24. K. Vogel, H. Risken, Phys. Rev. A 40, 2847-2849 (1989).

25. M. Cramer, et al., Nat. Comm., 1, 149, 2010.

26. H. Wolkowicz, G.P.H. Styan, Linear Algebra Appl. 29, 471-506, 1980.

27. K.G. Garren: Bounds for the Eigenvalues of a Matrix, NASA TN D-4373, 1968.

28. L.M. Delves, J. Phys. A: Gen. Phys., 5, 1972.

29. Löwdin P.-O. and Quantum Chemistry Group, Phys Rev, 139, A357, 1965.

30. J. Višñák, Integral error of the solution of the Schroedinger equation for selected systems, Bachelor Thesis, Mathematical-Physical Faculty, Charles University in Prague, (In Czech, 2007).

31. R.B. Griffiths, Ch-S. Niu, Phys. Rev. Lett. 76, No 17, 3228-3231 (1996).

32. J. Višňák, Quantum chemical algorithms for Quantum Computers, Diploma Thesis, Mathematical-Physical Faculty, Charles University in Prague (In Czech, 2012).

33. N. Hatano and M. Suzuki, Quantum annealing and other optimization methods, in Lecture Notes in Physics, chap. Finding Exponential Product Formulas of Higher Orders, Springer, Heidelberg, 2005.

34. S. Wiesner, arXiv:quant-ph/9603028v1 (1996).

35. C. Zalka, Proc. R. Soc. A 454, 313-322 (1998).

36. D.S. Abrams, S. Lloyd, Phys. Rev. Lett. 79, 25862589 (1997).

37. R. Babbush, J. McClean, D. Wecker, A. AspuruGuzik, N. Wiebe, Phys. Rev. A 91, 022311 (2015).

38. M-H. Yung, J.D. Whitfield, S. Boixo, D. Tempel, A. Aspuru-Guzik, Adv. Chem. Phys. 154 (2014).

39. M. Dobšíček, G. Johansson, V. Shumeiko, G. Wendin, Quantum Simulations: Report, Chalmers University, MC2 (2010).

40. T. Hamada, I.D. Johnston, Nucl. Phys. 34, 382-403 (1962).

41. R.B. Wiringa, V.G.J. Stoks, R. Schiavilla, Phys. Rev. C, 51, No 1 (1995).

42. S.N. Mosley, The positive radial momentum operator, arXiv:math-ph/0309055, (2003).

43. A. Klappenecker, M. Rötteler, Proc 2nd ISPA01, 37, 464-468, IEEE (2001).

44. P. Jordan, E. Wigner, Z. Phys., 47, Issue 9-10, pp 631-651 (1928).

45. S.B. Bravyi, A.Y. Kitaev, Ann. Phys. 298, Iss. 1, 210-226(2002).
46. J.T. Seeley, M.J. Richard, P.J. Love, J. Chem. Phys. 137, Iss. 22, p 224109 (2012).

47. R. Somma, G. Ortiz, E. Knill, J. Gubernatis, Int. J. Quantum Inform. 01, 189 (2003).

48. J. Višňák, L. Veis, J. Pittner, H. Nakai, Quantum chemistry beyond Born-Oppenheimer approximation on a quantum computer: a simulated phase estimation study (to be published).

49. J.R. McClean, R. Babbush, P.J. Love, A. AspuruGuzik, J. Phys. Chem. Lett., 5 Iss. 24, pp 4368-4380 (2014).

50. D. Wecker, B. Bauer, B.K. Clark, M.B. Hastings, M. Troyer, Phys. Rev. A 90, 022305 (2014).

51. D. Poulin, M.B. Hastings, D. Wecker, N. Wiebe, A.C. Doherty, M. Troyer, arXiv:1406.4920v1 (2014).

52. L. Veis, J. Pittner, J. Chem. Phys. 140, 214111 (2014)

53. J.-S. Xu, M.-H. Yung, X.-Y. Xu, S. Boixo, Z.-W. Zhou, Ch.-F. Li, A. Aspuru-Guzik, G.-C. Guo, Nat Photonics 8, 113-118, (2014).

54. D.W. Berry, A.M. Childs, Quantum Inf. Comput. 12, 29 (2012).

55. D.W. Berry, A.M. Childs, R. Cleve, R. Kothari, R.D. Somma, 46 ${ }^{\text {th }}$ STOC 2014 Proc., 283-292 (2014).

56. A. Peruzzo, J. McClean, P. Shadbolt, M-H. Yung, XQ. Zhou, P.J. Love, A. Aspuru-Guzik, J.L. O'Brien, Nat Comm, 5, 4213, (2014).

57. L. Hales, S. Hallgren, $41^{\text {st }}$ Proc. Ann. IEEE Symp (Comp Sci) Found, p. 515-525 (2000).

58. Wolfram Research, Inc., Mathematica, Version 10.0, Champaign, IL (2014).

59. http://www.naturalsession.wz.cz/kvantovka/Supplem entary Info QC nucl09.pdf

60. Y. Wang, et al., Quantum Simulation of Helium Hydride Cation in a Solid-State Spin Register, ACS Nano, (2015).

61. A. Günther, R. Roth, H. Hergert, S. Reinhardt, Phys. Rev. C 82, 024319 (2010). 